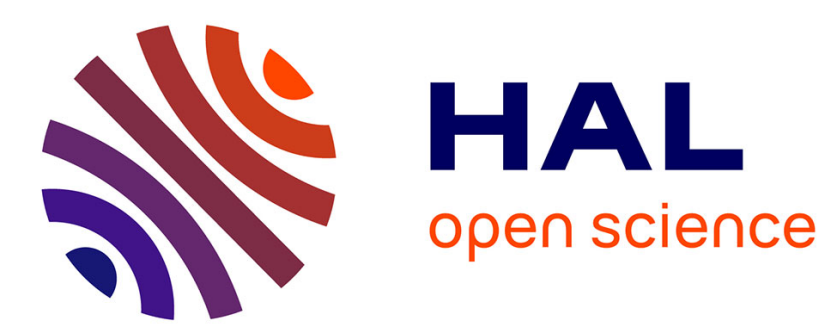

\title{
On parallel thinning algorithms: minimal non-simple sets, P-simple points and critical kernels
}

\author{
Gilles Bertrand, Michel Couprie
}

\section{To cite this version:}

Gilles Bertrand, Michel Couprie. On parallel thinning algorithms: minimal non-simple sets, P-simple points and critical kernels. Journal of Mathematical Imaging and Vision, 2009, 35 (1), pp.23-35. 10.1007/s10851-009-0152-3 . hal-00731784

\section{HAL Id: hal-00731784 \\ https://hal.science/hal-00731784}

Submitted on 13 Sep 2012

HAL is a multi-disciplinary open access archive for the deposit and dissemination of scientific research documents, whether they are published or not. The documents may come from teaching and research institutions in France or abroad, or from public or private research centers.
L'archive ouverte pluridisciplinaire HAL, est destinée au dépôt et à la diffusion de documents scientifiques de niveau recherche, publiés ou non, émanant des établissements d'enseignement et de recherche français ou étrangers, des laboratoires publics ou privés. 


\title{
On parallel thinning algorithms: minimal non-simple sets, $\mathbf{P}$-simple points and critical kernels
}

\author{
Gilles Bertrand and Michel Couprie \\ Université Paris-Est, Laboratoire d'Informatique Gaspard-Monge, Équipe A3SI, \\ ESIEE-Paris, France \\ e-mail: (g.bertrand,m.couprie)@esiee.fr
}

\begin{abstract}
Critical kernels constitute a general framework in the category of abstract complexes for the study of parallel homotopic thinning in any dimension. In this article, we present new results linking critical kernels to minimal nonsimple sets (MNS) and P-simple points, which are notions conceived to study parallel thinning in discrete grids. We show that these two previously introduced notions can be retrieved, better understood and enriched in the framework of critical kernels. In particular, we propose new characterizations which hold in dimensions 2, 3 and 4, and which lead to efficient algorithms for detecting $P$-simple points and minimal non-simple sets.
\end{abstract}

Key Words: Parallel thinning, topology preservation, critical kernel, P-simple point, minimal non-simple set, cubical complex, collapse, simple point, 4D space.

\section{Introduction}

Topology-preserving operators, such as homotopic skeletonization, are used in many applications of image analysis to transform an object while leaving unchanged its topological characteristics ${ }^{1}$. In discrete grids $\left(\mathbb{Z}^{2}, \mathbb{Z}^{3}, \mathbb{Z}^{4}\right)$, such a transformation can be defined thanks to the notion of simple point $[22,11]$ : intuitively, a point (or pixel in 2D, voxel in 3D) of an object is called simple if it can be deleted from this object while preserving topology. See for example Fig. 1a, where simple points are shown in gray.

The most "natural" way to thin an object consists of removing some of its border points in parallel, in a symmetrical manner. However, parallel deletion of simple points does not, in general, guarantee topology preservation: see for example Fig. 1a, where removing all simple points would split the object and merge two components of the

\footnotetext{
${ }^{1}$ To be more precise, we say that a transformation $\Psi$ "preserves topology" if $X$ is homotopy-equivalent to $\Psi(X)$ for any $X$.
}

background, and Fig. 1b,c,d,e where all the points are simple. In fact, such a guarantee is not obvious to obtain, even for the 2D case (see [10], where fifteen published parallel thinning algorithms are analyzed, and counter-examples are shown for five of them). In the 2D case, a popular method due to A. Rosenfeld [32] consists of dividing each thinning step into substeps. In each substep, only simple points that have no neighbor belonging to the object in one of the four main directions (north, south, east, west) are candidates for deletion. In addition, two special configurations made of two adjacent pixels (see Fig. 1c,d) must be preserved, as well as their $\pi / 2$ rotations. This stategy prevents a whole object to vanish like in Fig. 1b, or to break like in Fig. 1a. However, it cannot be straightforwardly extended to 3D. In this case, the six main directions are north, south, east, west, up and down. In Fig. 1e, the voxels $x, y$ are simple voxels that have no neighbor belonging to the object in the direction "up", but if we remove them in parallel, the object splits.

C. Ronse introduced minimal non-simple sets [31] to study the conditions under which points may be removed simultaneously while preserving topology of 2D objects. This leads to verification methods for the topological soundness of parallel thinning algorithms. Such methods have been proposed for 2D algorithms by C. Ronse [31] and R. Hall [14], they have been developed for the 3D case by T.Y. Kong [23, 17] and C.M. Ma [28], as well as for the 4D case by C-J. Gau and T.Y. Kong [12, 21]. Works dealing with 4D parallel homotopic thinning are indeed seldom, let us mention also the recent work of T.Y. Kong [20], and the work of A. Manzanera et al. [29]. For the 3D case, one of the authors [2] introduced the notion of P-simple point as a verification method but also as a methodology to design parallel thinning algorithms [3, 8, 26, 27].

Introduced recently by one of the authors, critical kernels [4] constitute a general framework in the category of abstract complexes for the study of parallel thinning in any dimension. Thanks to critical kernels, one can easily de- 
(a)

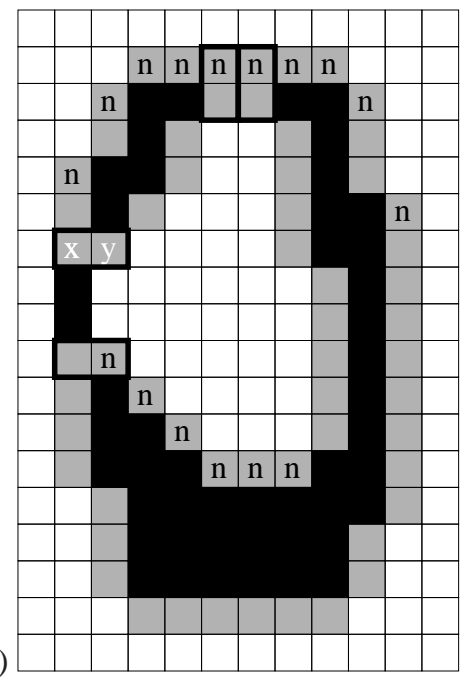

(b)

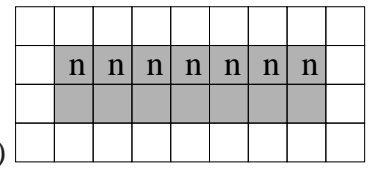

(d)

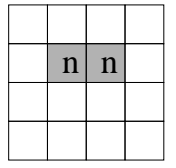

(c)

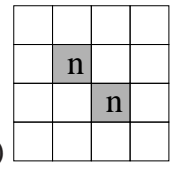

(e)

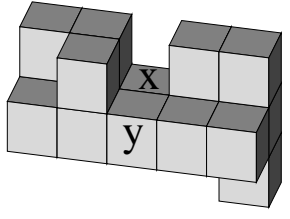

Figure 1. (a): The object is the set of all black and gray pixels. The pixels in gray are simple, but parallel removal of pixels $x, y$ for example would modify a topological characteristic of the object. North pixels are marked with the letter "n". (b,c,d): All pixels are simple. (e): All voxels are simple, the voxels $x$ and $y$ are both "up" voxels.

sign parallel thinning algorithms that produce new types of skeletons, with specific geometrical properties, while guaranteeing their topological soundness $[5,6]$. A new definition of a simple point is proposed in [4], based on the collapse operation which is a classical tool in algebraic topology and which guarantees topology preservation. Then, the notions of an essential face and of a core of a face are used to define the critical kernel $\mathcal{K}$ of a complex $X$. The most fundamental result proved in [4] is that, if a subset $Y$ of $X$ contains $\mathcal{K}$, then $X$ collapses onto $Y$, hence $X$ and $Y$ "have the same topology".

In this article, we show the equivalence (up to 4D) between the notion of P-simple point and a notion close to the one of crucial point, derived from the framework of critical kernels. This equivalence (Th. 21) leads to the first local

characterization of $\mathrm{P}$-simple points in 4D.

Furthermore, we show the equivalence (up to 4D) between the notion of MNS and the notion of crucial clique, also derived from the framework of critical kernels. This equivalence (Th. 27) leads to the first characterization of MNS which can be verified by using a polynomial method. We also retrieve straightforwardly some previously established properties of MNSs.

In order to ease the reading, proofs of new properties are deferred to the Appendix.

\section{Cubical Complexes}

Abstract complexes have been promoted in particular by V. Kovalevsky [25] and E. Khalimsky [16] in order to provide a sound topological basis for image analysis ${ }^{2}$. In this framework, we retrieve in particular the main notions and results of digital topology, such as the notion of simple point.

Intuitively, a cubical complex may be thought of as a set of elements having various dimensions (e.g. cubes, squares, edges, vertices) glued together according to certain rules. In this section, we recall briefly some basic definitions on complexes, see also [5, 6]. The way we define cubical complexes is purely discrete, whereas other authors adopt a continuous framework to define them (see e.g. [15]). We consider here $n$-dimensional complexes, with $0 \leq n \leq 4$.

Let $S$ be a set. If $T$ is a subset of $S$, we write $T \subseteq S$. We denote by $|S|$ the number of elements of $S$.

Let $\mathbb{Z}$ be the set of integers. We consider the families of sets $\mathbb{F}_{0}^{1}, \mathbb{F}_{1}^{1}$, such that $\mathbb{F}_{0}^{1}=\{\{a\} \mid a \in \mathbb{Z}\}, \mathbb{F}_{1}^{1}=\{\{a, a+$ 1\} $\mid a \in \mathbb{Z}\}$. A subset $f$ of $\mathbb{Z}^{n}, n \geq 2$, which is the Cartesian product of exactly $m$ elements of $\mathbb{F}_{1}^{1}$ and $(n-m)$ elements of $\mathbb{F}_{0}^{1}$ is called a face or an $m$-face of $\mathbb{Z}^{n}, m$ is the dimension of $f$, we write $\operatorname{dim}(f)=m$.

Observe that any non-empty intersection of faces is a face. For example, the intersection of two 2-faces $A$ and $B$ may be either a 2 -face (if $A=B$ ), a 1-face, a 0 -face, or the empty set.

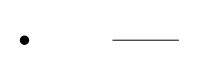

(a) (b)

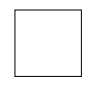

(c)

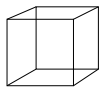

(d)

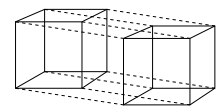

(e)
Figure 2. Graphical representations of: (a) a 0-face, (b) a 1-face, (c) a 2-face, (d) a 3-face, (e) a 4-face.

\footnotetext{
${ }^{2}$ An abstract complex is indeed a discrete topological space in the sense of P.S. Alexandroff [1].
} 
We denote by $\mathbb{F}^{n}$ the set composed of all $m$-faces of $\mathbb{Z}^{n}$, with $0 \leq m \leq n$. An $m$-face of $\mathbb{Z}^{n}$ is called a point if $m=0$, a (unit) edge if $m=1$, a (unit) square if $m=2$, a (unit) cube if $m=3$, a (unit) hypercube if $m=4$ (see Fig. 2).

Let $f$ be a face in $\mathbb{F}^{n}$. We set $\hat{f}=\left\{g \in \mathbb{F}^{n} \mid g \subseteq f\right\}$ and $\hat{f}^{*}=\hat{f} \backslash\{f\}$. Any $g \in \hat{f}$ is a face of $f$, and any $g \in \hat{f}^{*}$ is a proper face of $f$.

If $X$ is a finite set of faces in $\mathbb{F}^{n}$, we write $X^{-}=\cup\{\hat{f} \mid f \in$ $X\}, X^{-}$is the closure of $X$.

A set $X$ of faces in $\mathbb{F}^{n}$ is a cell or an $m$-cell if there exists an $m$-face $f \in X$, such that $X=\hat{f}$. The boundary of a cell $\hat{f}$ is the set $\hat{f}^{*}$.

A finite set $X$ of faces in $\mathbb{F}^{n}$ is a (discrete cubical) complex (in $\mathbb{F}^{n}$ ) if $X=X^{-}$. In other words, a complex is a union of cells. Any subset $Y$ of a complex $X$, which is also a complex, is a subcomplex of $X$. In Fig. 3, some complexes are represented.

Let $X \subseteq \mathbb{F}^{d}$ be a set of faces. A sequence $\pi=\left\langle f_{0}, \ldots, f_{\ell}\right\rangle$ of faces of $X$ is a path in $X$ (from $f_{0}$ to $f_{\ell}$ ) if either $f_{i}$ is included in $f_{i+1}$ or $f_{i+1}$ is included in $f_{i}$, for each $i \in$ $\{0, \ldots, \ell-1\}$.

Let $X \subseteq \mathbb{F}^{d}$. We say that $X$ is connected if, for any two faces $f, g$ in $X$, there is a path from $f$ to $g$ in $X$; otherwise we say that $X$ is disconnected. We say that $Y$ is a (connected) component of $X$ if $Y \neq \emptyset, Y \subseteq X, Y$ is connected and if $Y$ is maximal for these properties (i.e., we have $Z=Y$ whenever $Y \subseteq Z \subseteq X$ and $Z$ is connected). Notice that the empty set is connected but has no connected component.

Let $X \subseteq \mathbb{F}^{n}$. An $m$-face $f \in X$ is an $m$-facet of $X$, or simply a facet of $X$, if there is no $g \in X$ such that $f \in \hat{g}^{*}$.

Let $X$ be a complex in $\mathbb{F}^{n}, X \neq \emptyset$, the number $\operatorname{dim}(X)=$ $\max \{\operatorname{dim}(f) \mid f$ is a facet of $X\}$ is the dimension of $X$. We say that $X$ is an $m$-complex if $\operatorname{dim}(X)=m$.

We say that $X$ is pure if, for each facet $f$ of $X$, we have $\operatorname{dim}(f)=\operatorname{dim}(X)$.

In Fig. 3, the complexes (a) and (f) are pure, while (b,c,d,e) are not.

\section{Collapse and simple sets}

Intuitively a subcomplex of a complex $X$ is simple if its removal from $X$ preserves topology. In this section we recall a definition of a simple subcomplex based on the operation of collapse introduced by J.H.C. Whitehead ([33], see also $[13,9])$, which is a discrete analogue of a retraction, that is, a continuous deformation of an object onto itself.

Let $X$ be a complex in $\mathbb{F}^{n}$ and let $f \in X$. If there exists one face $g \in \hat{f}^{*}$ such that $f$ is the only face of $X$ that strictly includes $g$, then $g$ is said to be free for $X$ and the pair $(f, g)$ is said to be a free pair for $X$. Notice that, if $(f, g)$ is a free pair, then $f$ is necessarily a facet of $X$ and we have $\operatorname{dim}(g)=\operatorname{dim}(f)-1$.
Let $X$ be a complex. If $(f, g)$ is a free pair for $X$, then we say that there is an elementary collapse from $X$ to $X \backslash\{f, g\}$. Let $X, Y$ be two complexes. We say that $X$ collapses onto $Y$ if $Y=X$ or if there exists a collapse sequence from $X$ to $Y$, i.e., a sequence of complexes $\left\langle X_{0}, \ldots, X_{\ell}\right\rangle$ such that $X_{0}=X$, $X_{\ell}=Y$, and that there is an elementary collapse from $X_{i-1}$ to $X_{i}$, for all $i=1, \ldots, \ell$. If $X$ collapses onto $Y$ and $Y$ is a complex made of a single point, we say that $X$ is collapsible.

Fig. 3 illustrates a collapse sequence. Observe that, if $X$ is a cell of any dimension, then $X$ is collapsible. It may easily be seen that the collapse operation preserves the number of connected components. (a)

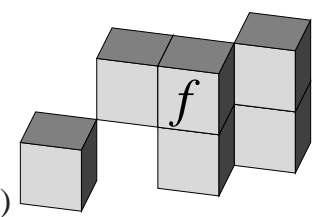

(c)

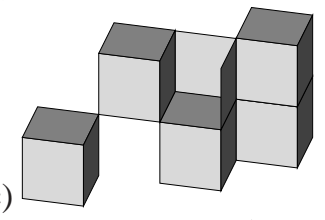

(e)

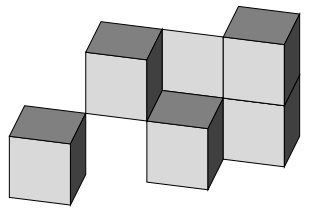

(b)

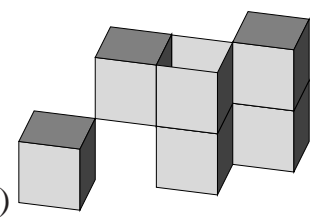

(d)

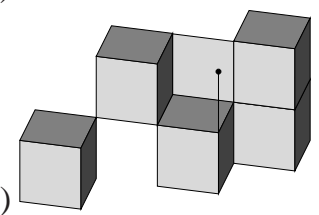

(f)

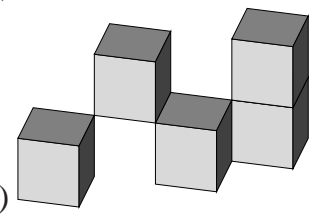

Figure 3. (a): A pure 3-complex $X$ in $\mathbb{F}^{3}$, and a 3-facet $f \in X$. (f): A complex $Y$ which is the detachment of $\hat{f}$ from $X$. (a-f): A collapse sequence from $X$ to $Y$.

Let $X, Y$ be two complexes. Let $Z$ such that $X \cap Y$ is a subcomplex of $Z$ and $Z$ is a subcomplex of $Y$, and let $f, g \in$ $Z \backslash X$. The pair $(f, g)$ is a free pair for $X \cup Z$ if and only if $(f, g)$ is a free pair for $Z$. Thus, by induction, we have the following property.

Proposition 1 ([4]). Let $X, Y$ be two complexes in $\mathbb{F}^{n}$. The complex $X \cup Y$ collapses onto $X$ if and only if $Y$ collapses onto $X \cap Y$.

The operation of detachment allows us to remove a subset from a complex, while guaranteeing that the result is still a complex.

Definition 2 ([4]). Let $X$ be a complex in $\mathbb{F}^{n}$ and let $Y \subseteq X$. We set $X \ominus Y=(X \backslash Y)^{-}$. The set $X \otimes Y$ is a complex which is the detachment of $Y$ from $X$.

In the following, we will be more particularly interested in the case where $Y$ is a single cell. For example in Fig. 3a, 
we see a complex $X$ containing a 3 -cell $\hat{f}$, and $X \ominus \hat{f}$ is depicted in Fig. 3f.

Let us now recall here a definition of simplicity [4] based on the collapse operation, which can be seen as a discrete counterpart of the one given by T.Y. Kong [18].

Definition 3 ([4]). Let $Y \subseteq X$; we say that $Y$ is simple for $X$ if $X$ collapses onto $X \otimes Y$.

The collapse sequence displayed in Fig. 3 (a-f) shows that the cell $\hat{f}$ is simple for the complex depicted in (a).

The notion of attachment, as introduced by T.Y. Kong $[17,18]$, leads to a local characterization of simple sets, which follows easily from Prop. 1.

Let $X$ be a complex in $\mathbb{F}^{n}$ and let $Y$ be a subcomplex of $X$. The attachment of $Y$ for $X$ is the complex defined by $\operatorname{Att}(Y, X)=Y \cap(X \otimes Y)$.

Proposition 4 ([4]). Let $X$ be a complex in $\mathbb{F}^{n}$ and let $Y$ be a subcomplex of $X$. The complex $Y$ is simple for $X$ if and only if $Y$ collapses onto $\operatorname{Att}(Y, X)$.

Let us recall two important properties proved in [11], which will be used to establish the main results of this paper. In [11], these properties where introduced for proving new characterizations of 2D, 3D and 4D simple points.

Consider three complexes $A, B, C$. If $A$ collapses onto $C$ and $A$ collapses onto $B$, then we know that $A, B$ and $C$ "have the same topology". If in addition we have $C \subseteq B \subseteq A$, it is tempting to conjecture that $B$ collapses onto $C$. Quite surprisingly this is not always true. Classical counter-examples to this assertion are Bing's house and the dunce hat ([7, 34], see also [11]).

For example, Bing's house can be obtained by collapse from a full cuboid (see Fig. 4). Nevertheless, it does not collapse onto any of its subsets: this object has no free pair. It is thus a counter-example for the above conjecture, with $A$ : a cuboid, $B$ : Bing's house, and $C$ : a point in $B$. In [11] we show that a dunce hat can also be realized as a cubical complex, and that it is also a counter-example for the same assertion.

In the two-dimensional discrete plane $\mathbb{F}^{2}$, such counterexamples cannot be found, consequently the above conjecture is true in this case. In [11] we show that, in the boundary of an $n$-face with $n \leq 4$, there is "not enough room" to build such counter-examples, and thus we have the two following properties.

Theorem 5 ([11]). Let $f$ be a $d$-face with $d \in\{2,3,4\}$, let $A, B$ be two subcomplexes of $\hat{f}^{*}$ such that $B \subseteq A$, and $A$ is collapsible. Then, $B$ is collapsible if and only if $A$ collapses onto $B$.

Theorem 6 ([11]). Let $f$ be a $d$-face with $d \in\{2,3,4\}$, and let $C, D$ be two subcomplexes of $\hat{f}^{*}$ such that $D \subseteq C$, and $\hat{f}$ collapses onto $D$. Then, $\hat{f}$ collapses onto $C$ if and only if $C$ (a)

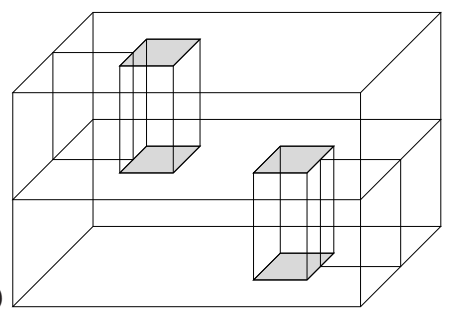

(b)

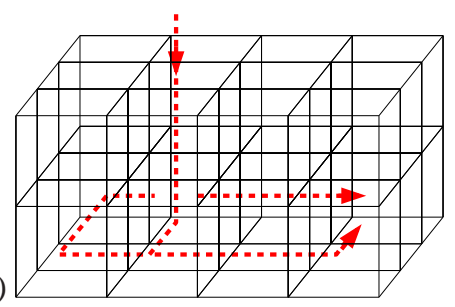

Figure 4. (a) Bing's house with two rooms (classical representation). The four rectangles in light gray are not part of the house, thus the lower room can be reached through the upper chimney, and vice-versa. (b) A 3complex made of 24 cubes. The arrows symbolize the order in which 3-collapse operations can be made in order to "carve" the lower room of the house. By performing a symmetrical operation for the upper room, we obtain a 2-complex (Bing's house) which has no free face.

collapses onto D.

It is also proved in [11] that extensions of Th. 5 and Th. 6 to dimension 5 and higher do not hold. This is due to the possibility to find, in a $d$-face with $d \geq 5$, counter-examples like Bing's house or the dunce hat.

Th. 7 and Cor. 8 (see below) constitute a key property ${ }^{3}$ which will be used to prove Prop. 19, Prop. 25 and Th. 21.

Theorem 7. Let $f$ be a d-face with $d \in\{2,3,4\}$, and let $X, Y$ be two subcomplexes of $\hat{f}^{*}$ such that $X$ is collapsible and $Y$ is collapsible. Then, $X \cup Y$ is collapsible if and only if $X \cap Y$ is collapsible.

Cor. 8 generalizes Th. 7 to an arbitrary number of subcomplexes.

Corollary 8. Let $f$ be a $d$-face with $d \in\{2,3,4\}$, let $\ell$ be an integer strictly greater than 1, let $X_{1}, \ldots, X_{\ell}$ be $\ell$ subcomplexes of $\hat{f}$. The two following assertions are equivalent:

i) For all $L \subseteq\{1, \ldots, \ell\}$ such that $L \neq \emptyset, \cup_{i \in L} X_{i}$ is collapsible.

\footnotetext{
${ }^{3}$ Notice that a similar property holds in $\mathbb{R}^{3}$, in the framework of algebraic topology, if we replace the notion of collapsibility onto a point by the one of contractibility $[19,30]$.
} 
ii) For all $L \subseteq\{1, \ldots, \ell\}$ such that $L \neq \emptyset, \cap_{i \in L} X_{i}$ is collapsible.

\section{Critical kernels}

Let us briefly recall the framework introduced by one of the authors (in [4]) for thinning, in parallel, discrete objects with the warranty that topology is preserved. We focus here on the two-, three- and four-dimensional cases, but in fact some of the results in this section are valid for complexes of arbitrary dimension. This framework is based solely on three notions: the notion of an essential face which allows us to define the core of a face, and the notion of a critical face.

Definition 9 ([4]). Let $X$ be a complex in $\mathbb{F}^{n}$ and let $f \in X$. We say that $f$ is an essential face for $X$ if $f$ is precisely the intersection of all facets of $X$ that contain $f$. We denote by $E s s(X)$ the set composed of all essential faces of $X$. If $f$ is an essential face for $X$, we say that $\hat{f}$ is an essential cell for $X$. If $Y$ is a subcomplex of $X$ and $\operatorname{Ess}(Y) \subseteq E$ ss $(X)$, then we say that $Y$ is an essential subcomplex of $X$.

Observe that a facet of $X$ is necessarily an essential face for $X$. Observe also that, if $X$ and $Y$ are both pure $n$ complexes, then $Y$ is an essential subcomplex of $X$ whenever $Y$ is a subcomplex of $X$.

Definition 10 ([4]). Let $X$ be a complex in $\mathbb{F}^{n}$ and let $f \in$ $\operatorname{Ess}(X)$. The core of $\hat{f}$ for $X$ is the complex Core $(\hat{f}, X)=$ $\cup\left\{\hat{g} \mid g \in \operatorname{Ess}(X) \cap \hat{f}^{*}\right\}$.

Definition 11 ([4]). Let $X$ be a complex in $\mathbb{F}^{n}$ and let $f \in X$. We say that $f$ and $\hat{f}$ are regular for $X$ if $f \in \operatorname{Ess}(X)$ and if $\hat{f}$ collapses onto Core $(\hat{f}, X)$. We say that $f$ and $\hat{f}$ are critical for $X$ if $f \in E s s(X)$ and if $f$ is not regular for $X$.

If $X$ be a complex in $\mathbb{F}^{n}$, we set Critic $(X)=\cup\{\hat{f} \mid f$ is critical for $X\}$, we say that $C r i t i c(X)$ is the critical kernel of $X$. $A$ face $f$ in $X$ is a maximal critical face, or an M-critical face (for $X$ ), if $f$ is a facet of $\operatorname{Critic}(X)$.

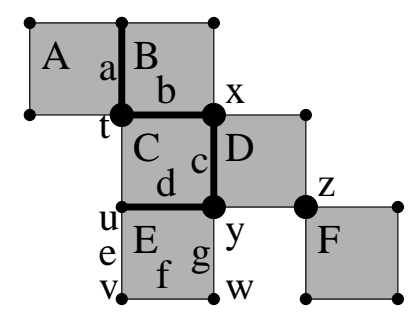

Figure 5. A complex $X$ for the illustration of the notions of essential face, core, critical and M-critical face (see text).
Fig. 5, where a complex $X$ is depicted, helps us to illustrate these notions. The letters $A, B, C, D, E, F$ indicate all the facets (2-faces), the letters $a, b, c, d, e, f, g$ indicate some of the 1-faces, and the letters $t, u, v, w, x, y, z$ some 0 -faces. The essential faces of the complex $X$ form the set $\operatorname{Ess}(X)=$ $\{A, B, C, D, E, F, a, b, c, d, t, x, y, z\}$ (essential 0-faces and 1faces are highlighted in the figure). The core of $\hat{D}$ for $X$ is the set $\{x, c, y, z\}$, the core of $\hat{E}$ for $X$ is $\{y, d, u\}$. We can see that a collapse sequence from the cell $\hat{E}$ to $\{y, d, u\}$ exists: e.g., $\langle\hat{E},\{v, f, w, g, y, d, u\},\{w, g, y, d, u\},\{y, d, u\}\rangle$. Thus, the facet $E$ is regular for $X$. On the other hand, we can see that no collapse sequence from $\hat{D}$ to $\{x, c, y, z\}$ exists, since the latter complex is disconnected: the facet $D$ is critical for $X$. We will see later that, as a consequence of Prop. 14, a facet is simple if and only if it is regular. Let us now consider the case of faces $a, b$ and $c$, which are not facets. We have $\operatorname{Core}(\hat{a}, X)=\{t\}$, Core $(\hat{b}, X)=\{t, x\}$ and $\operatorname{Core}(\hat{c}, X)=\{x, y\}$. Thus, $a$ is regular and $b, c$ are critical for $X$. An M-critical face is a face that is critical and not included in any other critical face, thus the face $b$ is M-critical (as it may be seen that both $B$ and $C$ are regular), but the face $c$ is not M-critical, since it is included in the critical face $D$. See also Fig. 6a for an illustration of critical faces in 3D.

The following properties of the core of a cell will be useful in the sequel.

Proposition 12 ([4]). Let $X$ be a complex in $\mathbb{F}^{n}$, and let $f \in \operatorname{Ess}(X)$. Let $K=\{g \in X \mid f \subseteq g\}$, and let $Y=X \odot K$. We have: Core $(\hat{f}, X)=\operatorname{Att}(\hat{f}, Y \cup \hat{f})=\hat{f} \cap Y$.

Corollary 13 ([4]). Let $X$ be a complex in $\mathbb{F}^{n}$, and let $f$ be a facet of $X$. We have: $\operatorname{Core}(\hat{f}, X)=\operatorname{Att}(\hat{f}, X)$.

As shown below, there is a strong link between the notions of regular and simple face.

Proposition 14 ([4]). Let $X$ be a complex in $\mathbb{F}^{n}$, and let $f \in \operatorname{Ess}(X)$. Let $K=\{g \in X \mid f \subseteq g\}$ and $Z=[X \otimes K] \cup \hat{f}$. The face $f$ is regular for $X$ if and only if $\hat{f}$ is simple for $Z$.

In particular, whenever $f$ is a facet, $f$ is regular for $X$ if and only if $f$ is simple for $X$ (since, in this case, we have $K=\{f\}$ and thus $Z=X$ ).

The following theorem is the most fundamental result concerning critical kernels. We will use it in the sequel of this paper in dimension 4 or less, but notice that the theorem holds whatever the dimension.

Theorem 15 ([4]). Let $n \in \mathbb{N}$, let $X$ be a complex in $\mathbb{F}^{n}$, and let $Y$ be an essential subcomplex of $X$.

i) The complex $X$ collapses onto its critical kernel.

ii) If $Y$ contains the critical kernel of $X$, then $X$ collapses onto $Y$.

iii) If $Y$ contains the critical kernel of $X$, and if $Z$ is an essential subcomplex of $X$ such that $Y \subseteq Z$, then $Z$ collapses onto $Y$. 


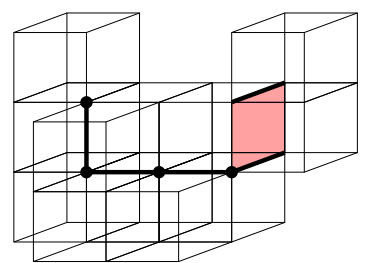

(a)

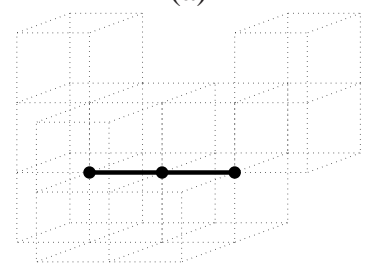

(c)

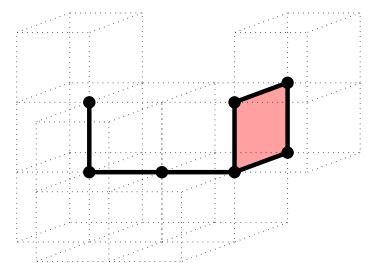

(b)

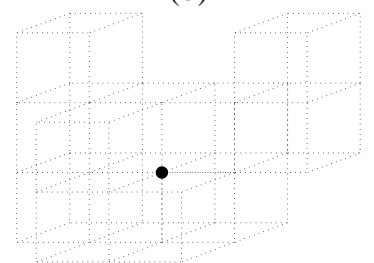

(d)
Figure 6. (a): A 3-complex $X$, made of 12 cubes, and its critical faces (highlighted). (b): The critical kernel $X_{1}=\operatorname{Critic}(X)$. (c): $X_{2}=$ $\operatorname{Critic}\left(X_{1}\right)$. (d): $X_{3}=\operatorname{Critic}\left(X_{2}\right)=\operatorname{Critic}\left(X_{3}\right)$.

In Fig. 6, we show that the very notion of critical kernel can be seen as a powerful thinning algorithm, which consists of computing iteratively the critical kernel of the preceding computation. Furthermore, Th. 15ii tells us that any essential subcomplex $Y$ of $X$ that is "between" $X$ (Fig. 6a) and $X_{1}$ (Fig. 6b) is such that $X$ collapses onto $Y$. This true, in particular, of any subcomplex $Y$ that is a pure 3-complex containing $X_{1}$. This property gives birth to a wide class of parallel thinning algorithms, where different criterions, based e.g. on geometrical notions, can be used in order to choose a particular set as the result of a single thinning step (see Sec. 4).

\section{Crucial kernels}

In the image processing literature, a digital image is often considered as a set of pixels in $2 \mathrm{D}$, voxels in $3 \mathrm{D}$, or 4-xels in 4D. A pixel (resp. voxel, 4-xel) is an elementary square (resp. cube, hypercube), thus an easy correspondence can be made between this classical view and the framework of cubical complexes. From now on, we consider only complexes that are unions of $d$-cells, i.e., pure $d$-complexes.

Notice that, if $X$ is a pure complex in $\mathbb{F}^{d}$ and if $\hat{f}$ is a $d$-cell of $X$, then $X \odot \hat{f}$ is a pure complex in $\mathbb{F}^{d}$. There is indeed an equivalence between the operation on complexes that consists of removing (by detachment) a simple $d$-cell, and the removal of an 8-simple (resp. 26-simple, 80-simple) point in the framework of 2D (resp. 3D, 4D) digital topology (see $[17,18])$.

If $X$ is a pure $d$-complex (e.g., a union of voxels in $\mathbb{F}^{3}$ ), the critical kernel of $X$ is not necessarily a pure $d$-complex (see Fig. 6b). The notion of crucial clique, introduced in [6], allows us to recover a pure $d$-subcomplex $Y$ of an arbitrary pure $d$-complex $X$, under the constraint that $X$ collapses onto $Y$.

Definition 16 ([6]). Let $X$ be a pure d-complex in $\mathbb{F}^{d}$, and let $f$ be an $M$-critical face for $X$. The set $K$ of all the facets of $X$ that contain $f$ is called a crucial clique (for $X$ ). More precisely, $K$ is the crucial clique induced by $f$.

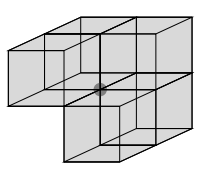

(a)

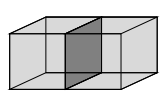

(d)

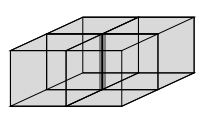

(b)

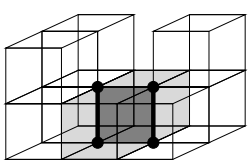

(e)

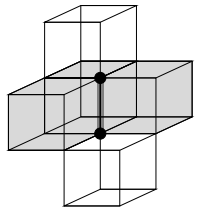

(c)

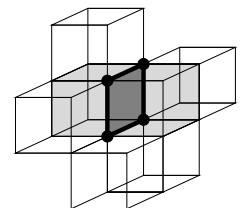

(f)
Figure 7. Crucial cliques in $\mathbb{F}^{3}$ (represented in light gray): (a) induced by an M-critical 0 -face; (b,c) induced by an M-critical 1-face; $(d, e, f)$ induced by an M-critical 2-face. The considered M-critical faces are in dark gray, the core of these M-critical faces (when nonempty) is represented in black.

Some 3D crucial cliques are illustrated in Fig. 7. Observe that Fig. 7e depicts precisely the configuration of voxels $x, y$ in Fig. 1e. It may be easily seen that, informally speaking, a thinning step that preserves all non-simple voxels and at least one voxel in each crucial clique, preserves topology.

The following parallel thinning scheme takes as input an "object" $X$ that is a pure $d$-complex, and a set $K$, called constraint set, composed of facets of $X$ that must be preserved during the thinning. 


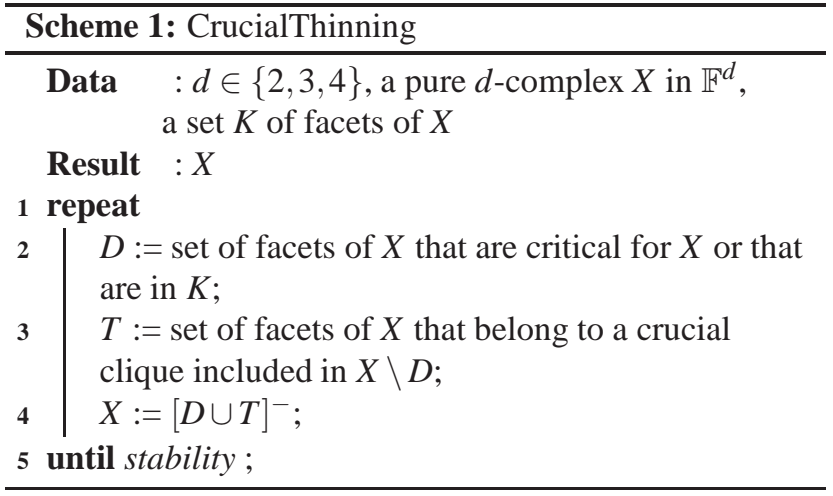

For each single step of Scheme 1, it may easily be seen that any critical face of $X$, if not contained in $K$ or in a critical facet of $X$, is contained in $T$. Thus, by Th. 15, we deduce that the set $X$ at step $i$ collapses onto the set $X$ at step $i+1$, and that Scheme 1 preserves topology.

Scheme 1 is very general, since any set $K$ may be used to constrain the thinning. Below, we give three examples of thinning methods based on this scheme, which illustrate the versatility of the critical kernels framework. Other examples may be found in $[5,6]$.

\section{Example 1: symmetrical thinning without constraint} set.

In Fig. 8 and Fig. 9, we illustrate the steps of Scheme 1 with no constraint $(K=\emptyset)$. The original objects are the same as in Fig. 1a and Fig. 1e respectively. In Fig. 8, three steps are needed to reach stability, and in Fig. 9 only two steps are necessary.

Such minimal skeletons may be used in some applications where we are not interested to keep the branches of a skeleton.

\section{Example 2: symmetrical thinning with medial axis.}

A ball $B$ is maximal for an object $X$ if $B$ is included in $X$ and is not strictly included in any other ball included in $X$. The set of all the centers of its maximal balls is often called the medial axis of the object.

In Fig. 10, we show an example of curvilinear skeleton in 2D and surface skeleton in 3D, obtained by the above parallel thinning scheme. The constraint set $\mathrm{K}$ is the medial axis based on the Manhattan distance in the 2D case, and the subset of the medial axis representing the locus of centers of maximal balls with radius greater than a chosen threshold (3) in the 3D case.

\section{Example 3: thinning with directional strategy.}

We have seen in the introduction that the directional strategy for parallel thinning, proposed by Rosenfeld in 2D [32], cannot be straightforwardly extended to the 3D case. In fact, the question of knowing whether this strategy
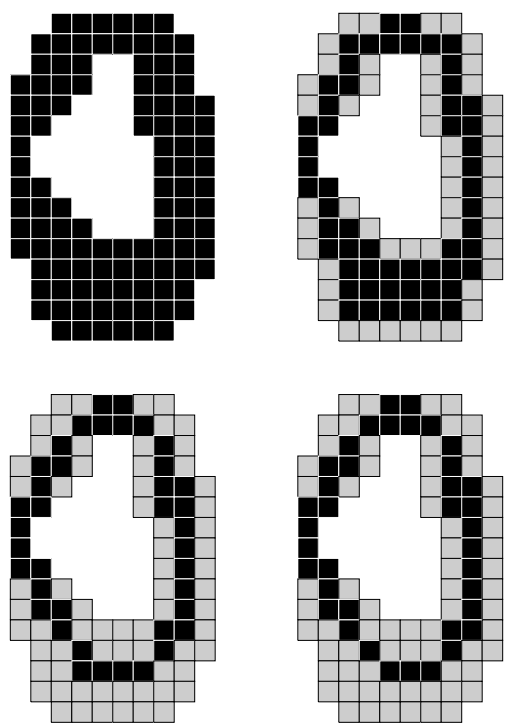

Figure 8. Illustration of Scheme 1 in 2D, $K=\emptyset$.
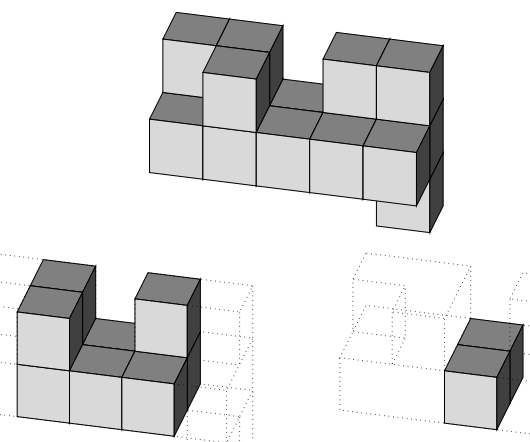

Figure 9. Illustration of Scheme 1 in 3D, $K=\emptyset$.

has a "natural extension" to 3D was cited among three open questions relative to digital topology by Kong, Litherland and Rosenfeld in [24] (question 547). We show here that the critical kernels framework indeed offers a direct way to extend the directional strategy to dimensions higher than 2 . A slight variant of Scheme 1 consists of computing the set $K$ dynamically, at each iteration, from the current state of the set $X$.

To implement in 3D the directional method, let us take as constraint $K$ the set of all points that are not "up" voxels, letting as candidates for deletion only those voxels that have no neighbor belonging to the object in the "up" direction: see the result in Fig. 11b. Repeating this procedure with down, north, south, east, and west voxels achieves one step of directional thinning, as illustrated in Fig. 11. Notice that no "special configuration" (like the ones of Fig. 1c,d) need 

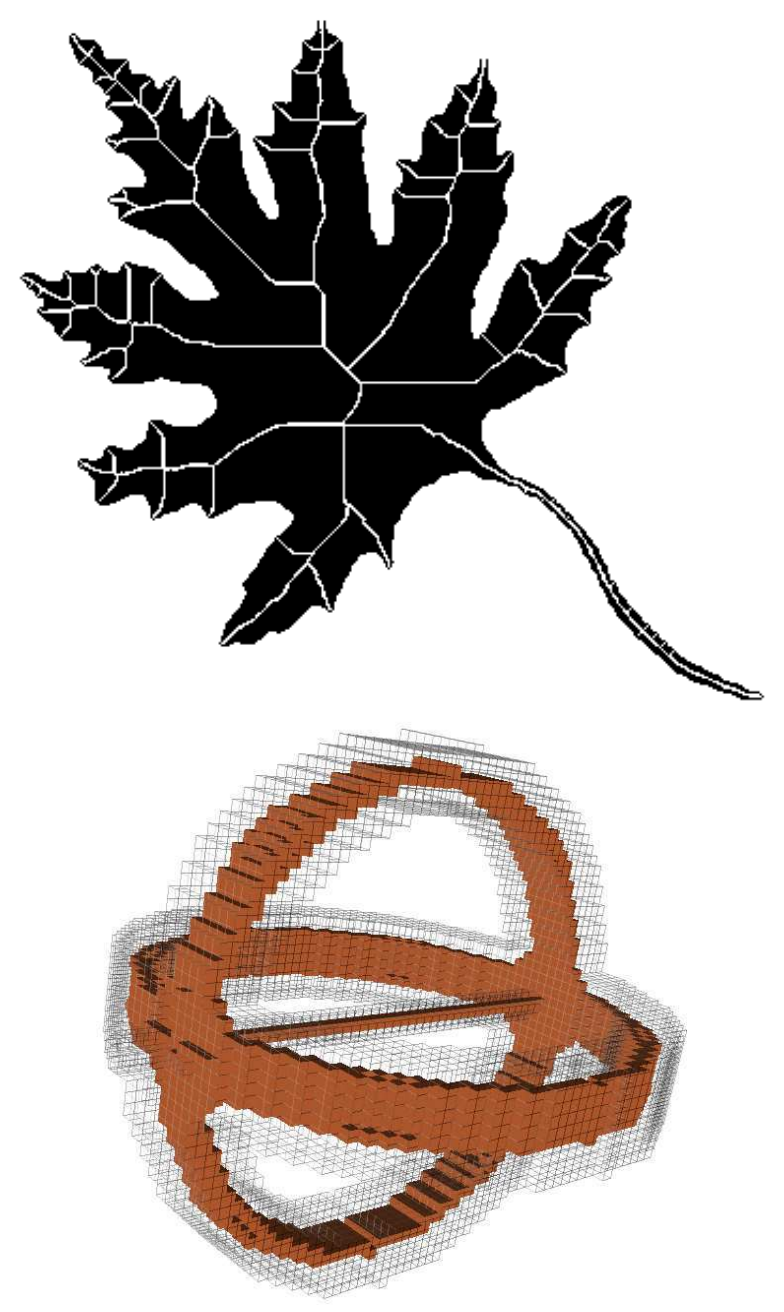

Figure 10. A 2D curvilinar skeleton (top), and a 3D surface skeleton (bottom), obtained thanks to the CrucialThinning scheme.

to be introduced, in 2D as in 3D. Such configurations are indeed crucial cliques, thus Scheme 1 automatically preserves them. Notice also that similar extensions may be done in dimensions higher than 3 .

In addition to these three examples, let us notice that by way of such constraint sets, some geometric conditions such as curve end or surface border detection may also be introduced $[5,6]$, both with the symmetrical and with the directional strategy. Let us mention that for the 3D case, a more powerful thinning scheme has been proposed in [5], based on a variant of the notion of crucial clique.

It should be noted that in 2D and 3D, the methodology based on crucial cliques does not need to handle the structure of abstract complexes. In fact, we showed in [6] that 2D crucial cliques may be characterized through a set of patterns defined in the classical square grid, as in most pa-
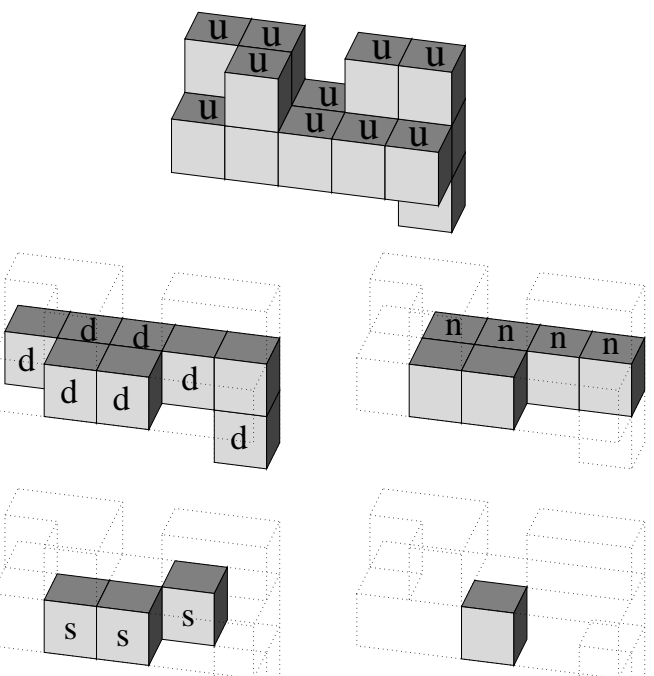

Figure 11. Directional thinning. Voxels marked $u, d, n, s$ are respectively up, down, north and south voxels.

pers related to parallel thinning.

Now, let us state two properties of crucial cliques which are essential for the proof of one of our main results (Th. 27).

Proposition 17. Let $X$ be a pure $d$-complex in $\mathbb{F}^{d}$, with $d \in$ $\{2,3,4\}$, let $f$ be an $M$-critical face of $X$, let $K$ be the crucial clique induced by $f$, and let $k$ be any facet of $K$. Let $K^{\prime}$ be such that $K^{\prime} \subseteq K \backslash\{k\}$ and $K^{\prime} \neq K \backslash\{k\}$.

Then, $k$ is a simple face of the complex $X \ominus K^{\prime}$.

Proposition 18. Let $X$ be a pure $d$-complex in $\mathbb{F}^{d}$, with $d \in$ $\{2,3,4\}$, let $f$ be an $M$-critical face of $X$, let $K$ be the crucial clique induced by $f$, and let $k$ be any facet of $K$.

Then, $k$ is not a simple face of the complex $[X \otimes K] \cup \hat{k}$.

Prop. 19, below, plays a role in the proofs of Prop. 25 (hence also Th. 27) and Th. 20.

Proposition 19. Let $X$ be a pure $d$-complex in $\mathbb{F}^{d}$, with $d \in$ $\{2,3,4\}$, let $K$ be a set of facets of $X$, let $k \in K$, such that $k$ is not simple for $[X \otimes K] \cup \hat{k}$ and $k$ is simple for $\left[X \otimes K^{\prime}\right]$ whenever $K^{\prime} \subseteq K \backslash\{k\}$ and $K^{\prime} \neq K \backslash\{k\}$. Then, $\cap\{h \in K\}$ is a face.

Some crucial cliques have a particularly simple structure: those which are reduced to a small connected component, where each element is adjacent to each other (like those in Fig. 7a,b,d). We show that those are precisely the crucial cliques that are induced by an M-critical face having an empty core.

Theorem 20. Let $X$ be a pure $d$-complex in $\mathbb{F}^{d}$, with $d \in$ 
$\{2,3,4\}$, let $K$ be a crucial clique for $X$ induced by an $M$ critical face $f$. Then, $K^{-}$is a connected component of $X$ if and only if $\operatorname{Core}(\hat{f}, X)=\emptyset$.

\section{$5 \quad$ P-simple points}

In the framework of digital topology, one of the authors introduced the notion of P-simple point [3]. P-simple points can be used as a verification method, but also as a methodology to design parallel thinning algorithms [3, 8, 26, 27].

We show in this section that there exists an equivalence between the notion of P-simple points and a notion derived from the one of crucial clique. This equivalence has been stated for the 2D case in [6], here we show that it extends up to $4 \mathrm{D}$.

First, we recall the definition of P-simple points. In this definition, the set $C$ is a set of points that are "candidates for deletion".

Let $X$ be a pure $d$-complex in $\mathbb{F}^{n}$, and let $C$ be a set of facets of $X$. A facet $k \in C$ is said to be $P$-simple for $\langle X, C\rangle$ if $k$ is simple for all complexes $X \ominus T$, such that $T \subseteq C \backslash\{k\}$.

For example, consider Fig. 1a and Fig. 1b, and assume in each case that $C$ is the set of all simple facets (in gray). Then, no facet in Fig. $1 \mathrm{~b}$ is P-simple, and all simple facets in Fig. 1a, except the ones that are delineated in bold, are $\mathrm{P}$-simple for $\langle X, C\rangle$.

The following parallel thinning scheme based on Psimple points takes as input, as CrucialThinning (see Sec. 4), an "object" $X$ which is a pure $d$-complex, and a set $K$, called constraint set, composed of facets of $X$ that must be preserved during the thinning.

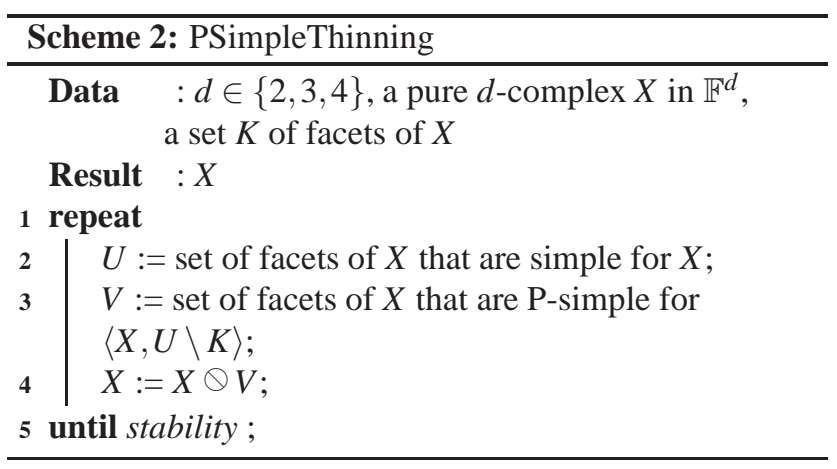

For the 3D case, a local characterization that leads to a linear algorithm for testing P-simplicity has been proved in [3]. Until now, such a characterization was not available in $4 \mathrm{D}$.

The following equivalence leads, in particular, to a local characterization and to the first algorithm for testing Psimplicity in 4D.

Theorem 21. Let $X$ be a pure $d$-complex in $\mathbb{F}^{d}$, with $d \in$ $\{2,3,4\}$, let $C$ be a set of facets of $X$, let $D=X \otimes C$. Let $k \in C$, the facet $k$ is $P$-simple for $\langle X, C\rangle$ if and only if $k$ does not contain any face $f$ that is critical for $X$, and such that all the facets of $X$ containing $f$ are in $C$.

Thus, any facet that is $\mathrm{P}$-simple for $\langle X, C\rangle$ is not crucial for $\langle X, D\rangle$. The converse is not true. For example in $\mathbb{F}^{2}$, if we consider a set $X$ that consists of a two-pixels width ribbon (see Fig. 1b), and if we denote by $C$ the set of all facets of $X$, it may be seen that the four pixels at the extremities of the ribbon are not crucial for $\langle X, D\rangle$, but also not P-simple for $\langle X, C\rangle$.

Indeed, it is possible to remove more facets with a thinning scheme that deletes simple facets that are not crucial than with one that deletes P-simple facets. In other words, the scheme CrucialThinning is more powerful than PSimpleThinning. To illustrate this, consider again the example of Fig. $1 \mathrm{~b}$, with no constraint set $(K=\emptyset)$. One step of CrucialThinning deletes four pixels, and three steps reduces the ribbon to just two pixels, while PSimpleThinning leaves this object unchanged.

Let $X$ be a pure $d$-complex in $\mathbb{F}^{d}$, and let $Y$ be a pure $d$-subcomplex of $X$. We say that $Y$ is a crucial retraction of $X$ if $Y$ contains all the critical facets of $X$, and at least one facet of each crucial clique for $X$. By Th. 15, if $Y$ is a crucial retraction of $X$ then $X$ collapses onto $Y$. In fact, despite the appearance, it is possible to check only with the notion of P-simple points whether the result of one step of a given algorithm is a crucial retraction or not. Since every critical face is included in an M-critical face, by Th. 21, we have the following.

Proposition 22. Let $X$ be a pure $d$-complex in $\mathbb{F}^{d}$, with $d \in$ $\{2,3,4\}$, let $T$ be a set of facets of $X$. Let $U$ be the set of all facets of $X$ that are not in $T$. The complex $T^{-}$is a crucial retraction of $X$ if and only if each element of $U$ is $P$-simple for $\langle X, U\rangle$.

\section{Minimal non-simple sets}

In the preceding section, we saw that critical kernels, which are settled in the framework of abstract complexes, allow us to retrieve the notion of P-simple point proposed in the context of digital topology. Now, we show that the notion of minimal non-simple set can also be retrieved in the framework of critical kernels.

C. Ronse introduced in [31] the minimal non-simple sets (MNS) to propose some conditions under which simple points can be removed in parallel while preserving topology. This leads to verification methods for the topological soundness of 2D thinning algorithms [31, 14], 3D thinning algorithms [23, 17, 28], the 4D case has even been considered in $[12,19,21]$.

The main result of this section (Th. 27) proves the equivalence between MNS and crucial cliques in dimensions 2, 3 
and 4. This equivalence leads to the first characterization of MNS which can be verified using a polynomial method. In contrast, the very definition of a MNS (see below), as well as the characterization of Th. 23, involves the examination of all subsets of a given candidate set, e.g., a subset of a $2 \times 2 \times 2 \times 2$ block in $4 \mathrm{D}$.

Let $X$ be a pure $d$-complex in $\mathbb{F}^{d}$, with $d \in\{2,3,4\}$. A sequence $\left\langle k_{0}, \ldots, k_{\ell}\right\rangle$ of facets of $X$ is said to be a simple sequence for $X$ if $k_{0}$ is simple for $X$, and if, for any $i \in$ $\{1, \ldots, \ell\}, k_{i}$ is simple for $X \otimes\left\{k_{j} \mid 0 \leq j<i\right\}$. Let $K$ be a set of facets of $X$. The set $K$ is said to be $F$-simple (where "F" stands for facet) for $X$ if $K$ is empty, or if the elements of $K$ can be ordered as a simple sequence for $X$. The set $K$ is minimal non-simple for $X$ if it is not F-simple for $X$ and if all its proper subsets are F-simple. The following characterization will be used in the sequel.

Theorem 23 (adapted from Gau and Kong [12], theorem 3). Let $X$ be a pure $d$-complex in $\mathbb{F}^{d}$, with $d \in\{2,3,4\}$, and let $K$ be a set of facets of $X$. Then $K$ is a minimal non-simple set for $X$ if and only if the two following conditions hold:

i) Each $k$ of $K$ is non-simple for $[X \otimes K] \cup \hat{k}$.

ii) Each $k$ of $K$ is simple for $\left[X \otimes K^{\prime}\right]$ whenever $K^{\prime} \subseteq K \backslash\{k\}$ and $K^{\prime} \neq K \backslash\{k\}$.

For example, it may be seen that the set $\{x, y\}$ in Fig. 1a, the set $\{x, y\}$ in Fig. 1e, as well as the sets displayed in Fig. 7 in light gray, are indeed minimal non-simple sets.

From Th. 23 and Prop. 19, we can directly retrieve a result previously established by Gau and Kong.

Theorem 24 (adapted from Gau and Kong [12], theorem 5). Let $X$ be a pure 4-complex in $\mathbb{F}^{4}$ (resp. 3-complex in $\mathbb{F}^{3}, 2$-complexin $\mathbb{F}^{2}$ ) and let $K$ be a set of facets of $X$. If $K$ is a minimal non-simple set for $X$, then $K$ is a non-empty subset of some $2 \times 2 \times 2 \times 2$ block of sixteen 4 -faces (resp. $2 \times 2 \times 2$ block of eight 3 -faces, $2 \times 2$ block of four 2 -faces).

Prop. 25 and Prop. 26 are steps for the proof of the main result of this section, Th. 27.

Proposition 25. Let $X$ be a pure d-complex in $\mathbb{F}^{d}$, with $d \in$ $\{2,3,4\}$, let $K$ be a minimal non-simple set for $X$, and let $f$ be the intersection of all the elements of $K$. Then, $f$ is a critical face for $X$.

Proposition 26. Let $X$ be a pure $d$-complex in $\mathbb{F}^{d}$, with $d \in$ $\{2,3,4\}$, let $K$ be a minimal non-simple set for $X$, and let $f$ be the intersection of all the elements of $K$. Then, $f$ is an $M$-critical face for $X$ and $K$ is the crucial clique induced by $f$.

If $K$ is a crucial clique for $X$, then from Th. 23, Prop. 17 and Prop. $18, K$ is a minimal non-simple set for $X$. Conversely, if $K$ is a minimal non-simple set for $X$, then by Prop. 26, $K$ is a crucial clique. Thus, we have the following theorem.
Theorem 27. Let $X$ be a pure $d$-complex in $\mathbb{F}^{d}$, with $d \in$ $\{2,3,4\}$, and let $K$ be a set of facets of $X$. Then $K$ is a minimal non-simple set for $X$ if and only if it is a crucial clique for $X$.

We retrieve thanks to Th. 20, and by inspection of all possible configurations, a previously established property about MNSs that are connected components [31, 14, 17, 19], its most general formulation being found in [19].

Proposition 28 (adapted from Kong [19], theorem 3-2). Let $X$ be a pure $d$-complex in $\mathbb{F}^{d}$, with $d \in\{2,3,4\}$, let $K$ be a set of facets of $X$, such that $K^{-}$is a connected component of $X$, and such that the intersection of all its facets is non-empty. Then, $K$ is a minimal non-simple set for $X$.

We also retrieve thanks to Th. 20, and from the observation that a face with a non-empty core has necessarily a dimension that is at least 1 , the following property.

Proposition 29 (adapted from Gau and Kong [12], theorem 8-2). Let $X$ be a pure 4-complex in $\mathbb{F}^{4}$ (resp. 3-complex in $\mathbb{F}^{3}, 2$-complex in $\left.\mathbb{F}^{2}\right)$, let $K$ be a MNS such that $K^{-}$is not a connected component of $X$. Then, $K$ is a non-empty subset of a $2 \times 2 \times 2$ block of eight 4 -faces (resp. a $2 \times 2$ block of four 3-faces, a set of two 2-faces having an intersection that is a 1 -face).

\section{Conclusion}

Critical kernels constitute a powerful framework to study parallel homotopic thinning in any dimension. Indeed, the very notion of critical kernel may be seen as thinning scheme, which consists of iteratively computing the critical kernel of the result of the previous step. Critical kernels may also be used to design new algorithms, as well as to check the topological validity of existing ones.

We demonstrated in this article that the main concepts previously introduced in order to study topology-preserving parallel thinning in the framework of digital topology, namely P-simple points and minimal non-simple sets, may be not only retrieved in the framework of critical kernels, but also better understood and enriched. Critical kernels thus appear to constitute a unifying framework which encompasses previous works on parallel thinning.

Furthermore, in contrast with minimal non-simple sets, critical kernels provide a methodology to produce thinning algorithms which preserve topology "by construction", and we showed in this paper that these algorithms are more powerful than those which may be designed on the basis of Psimple points.

\section{Appendix}

In the sequel, we denote by $X^{+}$the set of all facets of a complex $X$. 
Proof of Th. 7. Suppose that $X \cup Y$ is collapsible. By applying Th. 5 with $A=X \cup Y$ and $B=X$, we deduce that $X \cup Y$ collapses onto $X$. By Prop. 1, we deduce that $Y$ collapses onto $X \cap Y$. And by applying Th. 5 with $A=Y$ and $B=X \cap Y$, we deduce that $X \cap Y$ is collapsible.

Now, suppose that $X \cap Y$ is collapsible. By applying Th. 5 with $A=X$ and $B=X \cap Y$, we deduce that $X$ collapses onto $X \cap Y$. By Prop. 1, we deduce that $X \cup Y$ collapses onto $Y$. And since $Y$ is collapsible, by transitivity $X \cup Y$ is collapsible.

Proof of Cor. 8. By Th. 7, the property holds whenever $\ell=2$. Suppose that it holds until $\ell-1 \geq 2$, and let us prove it for $\ell$.

$\mathrm{i} \Rightarrow$ ii. For any $j \in\{1, \ldots, \ell\}$, let $Z_{j}=\cap_{i=1}^{i=j} X_{i}$. For any $L \subseteq\{1, \ldots, \ell\}, 0<|L|<\ell$, the recurrence hypothesis implies that $\cap_{i \in L} X_{i}$ is collapsible, in particular $Z_{\ell-1}$ is collapsible. It remains to prove that $Z_{\ell}$ is collapsible. For any $j \in\{1, \ldots, \ell-1\}$, let $Y_{j}=X_{j} \cup X_{\ell}$. For all $L \subseteq\{1, \ldots, \ell-1\}$, $L \neq \emptyset$, we see that $\cup_{i \in L} Y_{i}$ is collapsible. From the recurrence hypothesis, we deduce that $\cap_{i=1}^{i=\ell-1} Y_{i}$ is collapsible. But, $\cap_{i=1}^{i=\ell-1} Y_{i}=Z_{\ell-1} \cup X_{\ell}$. Since $Z_{\ell-1} \cup X_{\ell}, Z_{\ell-1}$ and $X_{\ell}$ all are collapsible, from Th. 7 we deduce that $Z_{\ell-1} \cap X_{\ell}=Z_{\ell}$ is collapsible.

ii $\Rightarrow$ i. The proof is obtained by exchanging intersection and union in the previous reasoning.

Proof of Prop. 17. Let $Z=\left[X \odot K^{\prime}\right]$ and $Y=\left[X \otimes K^{\prime}\right] \otimes \hat{k}$ (see an illustration in Fig. 12). By definition of a crucial clique, the face $f$ is included in any facet of $K$, thus $f$ is contained in both $Z$ and $Y$. Suppose that $g$ is also an M-
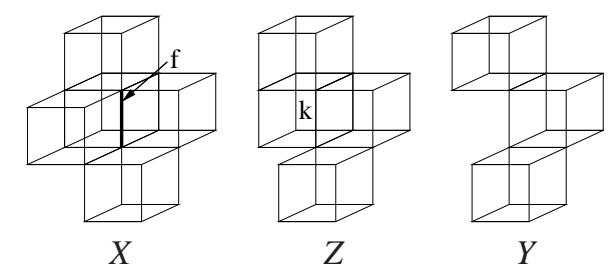

Figure 12. Illustration (in $\mathbb{F}^{3}$ ) for the proof of Prop. 17.

critical face for $X$ which is in $\left[K^{\prime}\right]^{-}$, and let $K^{\prime \prime}$ be the crucial clique for $X$ induced by $g$. If $K^{\prime \prime} \nsubseteq\left[K^{\prime} \cup\{k\}\right]$, then by definition of $Y, g$ must be included in a facet which is in $Y$. Otherwise $K^{\prime \prime} \subseteq K$, hence $f \subseteq g$, and by definition of an M-critical face, $f=g$. From this we see that $Y$ contains the critical kernel of $X$. Furthermore, $Z$ is an essential subcomplex of $X$ (since $Z^{+} \subseteq X^{+} \subseteq E s s(X)$ ) and $Y$ is an essential subcomplex of $X$ (idem). From Th. 15iii, we deduce that $Z$ collapses onto $Y$, in other words, $k$ is simple for $Z$.

Proof of Prop. 18. Let $W=[X \otimes K], Y=W \cup \hat{k}$, and $Z=W \cup \hat{f}$ (see an illustration in Fig. 13). We know that $Y$

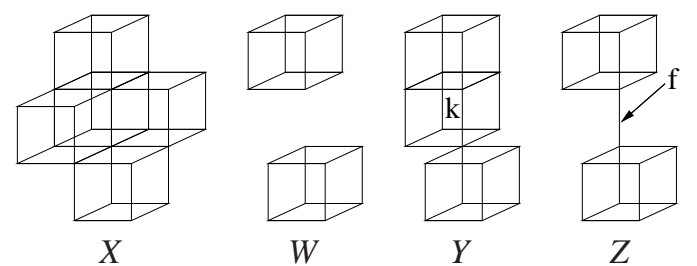

Figure 13. Illustration (in $\mathbb{F}^{3}$ ) for the proofs of Prop. 18 and Prop. 25.

is an essential subcomplex of $X$ (since $Y^{+} \subseteq X^{+} \subseteq \operatorname{Ess}(X)$ ) and we can see that $Z$ is an essential subcomplex of $X$, since $f$ is M-critical, thus essential for $X$. From Th. 15, we deduce that $Y$ collapses onto $Z$, and by Prop. 1, $\hat{k}$ collapses onto $\hat{k} \cap Z$ (i).

Suppose that $k$ is simple for $Y$, that is, $Y$ collapses onto $Y \otimes \hat{k}=W$, thus by Prop. $1, \hat{k}$ collapses onto $\hat{k} \cap W$ (ii). By definition of $Z$ and $W, \hat{k} \cap W$ is a subcomplex of $\hat{k} \cap Z$. Thus from (i), (ii) and Th. 6, we deduce that $\hat{k} \cap Z$ collapses onto $\hat{k} \cap W$.

Remark that $\hat{k} \cap Z=[\hat{k} \cap W] \cup \hat{f}$, thus by Prop. $1, \hat{f}$ collapses onto $\hat{f} \cap[\hat{k} \cap W]=\hat{f} \cap W$. Again by Prop. 1, we deduce that $Z=W \cup \hat{f}$ collapses onto $W$, i.e., $f$ is simple for $Z$, a contradiction with Prop. 14 and the fact that $f$ is critical for $X$. $\square$

Proof of Prop. 19. Notice that the property trivially holds when $|K|=1$, suppose now that $|K|=\ell>1$. Let us write $K=\left\{k=k_{1}, k_{2}, \ldots, k_{\ell}\right\}$. By hypothesis, $k$ is simple for $\cup_{i \in L}\left\{[X \otimes K] \cup \hat{k} \cup \hat{k}_{i}\right\}, \forall L \subseteq\{2, \ldots, \ell\}$ such that $L \neq \emptyset$. By Prop. 4, $\hat{k}$ collapses onto $\cup_{i \in L}\left\{[X \otimes K] \cup \hat{k}_{i}\right\} \cap \hat{k}$ $=\cup_{i \in L}\left\{\left([X \otimes K] \cup \hat{k}_{i}\right) \cap \hat{k}\right\}, \forall L \subseteq\{2, \ldots, \ell\}$ such that $L \neq$ Ø. By Cor. 8 and Th. 5 (with $A=\hat{k}$ ), $\hat{k}$ collapses onto $\cap_{i=2}^{i=\ell}\left\{\left([X \otimes K] \cup \hat{k}_{i}\right) \cap \hat{k}\right\}=([X \otimes K] \cap \hat{k}) \cup\left(\left[\cap i=2 \hat{k}_{i}\right] \cap \hat{k}\right)=$ $([X \otimes K] \cap \hat{k}) \cup \hat{f}$, where $f=\cap\{h \in K\}$. Thus $f$ cannot be empty, otherwise $\hat{k}$ would collapse onto $[X \otimes K] \cap \hat{k}$, a contradiction with Prop. 4 and the fact that $k$ is not simple for $[X \otimes K] \cup \hat{k}$. Since $f$ is a non-empty intersection of faces, $f$ is a face.

Proof of Th. 20. Let us denote by $|C(Z)|$ the number of connected components of any set of faces $Z$. The property is straightforward when $|K|=1$, suppose now that $|K|>1$. Let $Y=X \otimes K$. Suppose that $K^{-}$is a connected component of $X$, thus $K^{-} \cap Y=\emptyset$. Since $\hat{f} \subseteq K^{-}$, we have $\hat{f} \cap Y=\emptyset$. By Prop. 12, $\hat{f} \cap Y=\operatorname{Core}(\hat{f}, X)$, hence $\operatorname{Core}(\hat{f}, X)=\emptyset$. Conversely, suppose that $\operatorname{Core}(\hat{f}, X)=\emptyset$, hence $\hat{f} \cap Y=\emptyset$ and $|C(Y \cup \hat{f})|=|\mathcal{C}(Y)|+1$. Since $f$ is M-critical for $X$, the critical kernel of $X$ is contained in $Y \cup \hat{f}$, furthermore $Y \cup \hat{f}$ is an essential subcomplex of $X$. By Th. 15ii, $X$ collapses onto $Y \cup \hat{f}$, hence $|\mathcal{C}(Y \cup \hat{f})|=|\mathcal{C}(X)|=|\mathcal{C}(Y)|+1$, and by definition of $Y, K^{-}$is a connected component of $X$.

The proof of Th. 21 will be given after the one of Prop. 25 for a more comfortable reading, as the two proofs 
share a common argument.

Proof of Prop. 25. From Th. 23 and Prop. 19, we deduce that $f$ is a face of $X$. Let $\ell=|K|$, if $\ell=1$ the property follows straightforwardly from the definitions. From now, suppose that $\ell \geq 2$. Let us write $K=\left\{k=k_{1}, k_{2}, \ldots, k_{\ell}\right\}$, $W=[X \odot K], Y=W \cup \hat{k}$, and $Z=W \cup \hat{f}$ (see Fig. 13 for an illustration). Let $L$ be any non-empty subset of $\{2, \ldots, \ell\}$, and let us write $Y_{L}=W \cup\left\{k_{i} \mid i \in L\right\}^{-}$. From Th. 23ii, $k$ is simple for $Y_{L} \cup \hat{k}$, that is, $Y_{L} \cup \hat{k}$ collapses onto $Y_{L}$. From Prop. 1, $\hat{k}$ collapses onto $\hat{k} \cap Y_{L}=\hat{k} \cap\left[W \cup\left\{k_{i} \mid i \in L\right\}^{-}\right]$ $=\hat{k} \cap\left[\cup\left\{W \cup \hat{k}_{i} \mid i \in L\right\}\right]=\cup\left\{\left[W \cup \hat{k}_{i}\right] \cap \hat{k} \mid i \in L\right\}$. From Cor. 8 and Th. 5 (with $A=\hat{k}$ ), we deduce that $\hat{k}$ collapses onto $\cap\left\{\left[W \cup \hat{k}_{i}\right] \cap \hat{k} \mid 2 \leq i \leq \ell\right\}=\hat{k} \cap\left[\cap\left\{W \cup \hat{k}_{i} \mid 1 \leq i \leq \ell\right\}\right]$ $=\hat{k} \cap Z$; and by Prop. $1, \bar{Y}=\hat{k} \cup Z$ collapses onto $Z$. Suppose that $f$ is not a critical face for $X$, i.e., $\hat{f}$ collapses onto $\operatorname{Core}(\hat{f}, X)$. From Prop. 12, we have $\operatorname{Core}(\hat{f}, X)=$ $\hat{f} \cap W$. Thus, since $Y$ collapses onto $W \cup \hat{f}$ and $\hat{f}$ collapses onto $\hat{f} \cap W$ (hence by Prop. $1 W \cup \hat{f}$ collapses onto $W$ ), we deduce that $Y$ collapses onto $W$, which means that $k$ is simple for $Y$, a contradiction with Th. 23i.

Proof of Th. 21. Suppose that $k$ is P-simple for $\langle X, C\rangle$. Let $f$ be any face in $k$ such that $f \neq k$ and such that the set $K$ of all facets of $X$ containing $f$ is included in $C$. Let us write $K=\left\{k=k_{1}, k_{2}, \ldots, k_{\ell}\right\}$. By definition of a P-simple facet, we know that $k$ is simple for $[X \ominus K] \cup \hat{k}$, thus by Prop. $4, \hat{k}$ collapses onto $[X \odot K] \cap \hat{k}(1)$. We also know that $k$ is simple for $\cup_{i \in L}\left\{[X \odot K] \cup \hat{k} \cup \hat{k}_{i}\right\}, \forall L \subseteq\{2, \ldots, \ell\}$ such that $L \neq \emptyset$. By Prop. 4, $\hat{k}$ collapses onto $\cup_{i \in L}\left\{[X \odot K] \cup \hat{k_{i}}\right\} \cap \hat{k}, \forall L \subseteq$ $\{2, \ldots, \ell\}$ such that $L \neq \emptyset$. By Cor. 8 and Th. $5, \hat{k}$ collapses onto $\cap i=2\left\{[X \ominus K] \cup \hat{k}_{i}\right\} \cap \hat{k}=(\hat{k} \cap[X \odot K]) \cup\left(\cap_{i=2}^{i=\ell}\left\{\hat{k_{i}} \cap \hat{k}\right\}\right)$ $=([X \odot K] \cap \hat{k}) \cup \hat{f}(2)$. Furthermore, $\hat{f}$ is collapsible (3) since $f$ is a cell. From (1), (2), (3), Th. 7 and Th. 5, we deduce that $\hat{k}$ collapses onto $([X \otimes K] \cap \hat{k}) \cap \hat{f}=[X \otimes K] \cap \hat{f}$, hence $[X \odot K] \cap \hat{f}$ is collapsible (by Th. 5), and $\hat{f}$ collapses onto $[X \otimes K] \cap \hat{f}$ (again by Th. 5). Thus by Prop. 14, $f$ is not critical for $X$. Since this holds for any such face $f$, the forward implication is proved.

Suppose now that $k$ is not $\mathrm{P}$-simple for $\langle X, C\rangle$. Thus, there exists a set $T \subseteq C \backslash\{k\}$ such that $k$ is not simple for $X \ominus T$. Without loss of generality, we suppose that $T$ is minimal for this property, i.e., $k$ is simple for $X \ominus T^{\prime}$ whatever the set $T^{\prime}$ strictly included in $T$. Let $f$ denote the intersection of all the facets of $T \cup\{k\}$. From Prop. 19, we deduce that $f$ is a face of $X$. From now on, the proof is essentially the same as the proof of Prop. 25, showing that $f$ is a critical face. $\square$

Proof of Prop. 26. See Fig. 14 for an illustration. From Prop. 25 we know that $f$ is critical for $X$, suppose that there exists a critical face $f^{\prime}$ for $X$ which strictly contains $f$. We may assume that $f^{\prime}$ is M-critical for $X$ (otherwise there would exist an M-critical face containing both $f^{\prime}$ and $f$, and this face should be chosen). Let $K^{\prime}=\left\{k \in X \mid f^{\prime} \subseteq k\right\}$ (by construction we have $K^{\prime} \subseteq K$ ), $Y=\left[X \odot K^{\prime}\right]$, and $Y^{\prime}=$
$Y \cup \hat{f}^{\prime}$. We have $K^{\prime} \neq K$, otherwise we would have $f^{\prime}=f$. Let $k \in K^{\prime}$ and let $Y^{\prime \prime}=Y \cup \hat{k}$. From Th. 23ii we deduce that
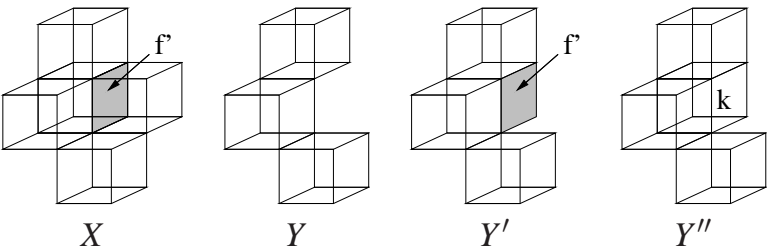

Figure 14. Illustration (in $\mathbb{F}^{3}$ ) for the proof of Prop. 26.

$Y^{\prime \prime}$ collapses onto $Y$. By Prop. 1, it follows that $\hat{k}$ collapses onto $\hat{k} \cap Y$ (1). The complex $Y^{\prime}$ contains, by construction, the critical kernel of $X$. Furthermore, since it can be seen that both $k$ and $f^{\prime}$ are essential for $X$, we know that $Y^{\prime \prime}$ is an essential subcomplex of $X$ and $Y^{\prime}$ is an essential subcomplex of $X$. By Th. 15iii, we deduce that $Y^{\prime \prime}$ collapses onto $Y^{\prime}$. By Prop. 1, it follows that $\hat{k}$ collapses onto $\hat{k} \cap Y^{\prime}$ (2). From (1), (2) and Th. 6, we deduce that $\hat{k} \cap Y^{\prime}$ collapses onto $\hat{k} \cap Y$, i.e., $(\hat{k} \cap Y) \cup \hat{f}^{\prime}$ collapses onto $\hat{k} \cap Y$. By Prop. 1, $\hat{f}^{\prime}$ thus collapses onto $(\hat{k} \cap Y) \cap \hat{f}^{\prime}=Y \cap \hat{f}^{\prime}$ and by Prop. 12 , $Y \cap \hat{f}^{\prime}=\operatorname{Core}\left(\hat{f}^{\prime}, X\right)$, hence a contradiction with the initial assumption that $f^{\prime}$ is critical for $X$.

\section{References}

[1] P.S. Alexandroff. Diskrete Räume. Math. Sbornik, 2(3):501-518, 1937.

[2] G. Bertrand. On P-simple points. Comptes Rendus de l'Académie des Sciences, Série Math., I(321):1077_ 1084, 1995.

[3] G. Bertrand. Sufficient conditions for 3D parallel thinning algorithms. In SPIE Vision Geometry IV, volume 2573, pages 52-60, 1995.

[4] G. Bertrand. On critical kernels. Comptes Rendus de l'Académie des Sciences, Série Math., I(345):363367, 2007.

[5] G. Bertrand and M. Couprie. A new 3D parallel thinning scheme based on critical kernels. In Discrete Geometry for Computer Imagery, volume 4245 of Lecture Notes in Computer Science, pages 580 591. Springer, 2006.

[6] G. Bertrand and M. Couprie. Two-dimensional parallel thinning algorithms based on critical kernels. Journal of Mathematical Imaging and Vision, 31(1):35-56, 2008. 
[7] R.H. Bing. Some aspects of the topology of 3manifolds related to the Poincaré conjecture. Lectures on modern mathematics, II:93-128, 1964.

[8] J. Burguet and R. Malgouyres. Strong thinning and polyhedric approximation of the surface of a voxel object. Discrete Applied Mathematics, 125:93-114, 2003.

[9] Marshall M. Cohen. A Course in Simple Homotopy Theory. Springer, 1973.

[10] M. Couprie. Note on fifteen $2 \mathrm{~d}$ parallel thinning algorithms. Internal Report, Université de Marne-laVallée, IGM2006-01, 2006.

[11] Michel Couprie and Gilles Bertrand. New characterizations of simple points in 2D, 3D and 4D discrete spaces. IEEE Trans. on Pattern Analysis and Machine Intelligence, 31(4):637-648, April 2009.

[12] C-J. Gau and T.Y. Kong. Minimal non-simple sets in 4D binary images. Graphical Models, 65:112-130, 2003.

[13] P. Giblin. Graphs, Surfaces and Homology. Chapman and Hall, 1981.

[14] R.W. Hall. Tests for connectivity preservation for parallel reduction operators. Topology and its Applications, 46(3):199-217, 1992.

[15] T. Kaczynski, K. Mischaikow, and M. Mrozek. Computational Homology. Springer, 2004.

[16] E. Khalimsky, R. Kopperman, and P.R. Meyer. Computer graphics and connected topologies on finite ordered sets. Topology and its Applications, 36:1-17, 1990.

[17] T. Y. Kong. On topology preservation in 2-D and 3-D thinning. International Journal on Pattern Recognition and Artificial Intelligence, 9:813-844, 1995.

[18] T. Y. Kong. Topology-preserving deletion of 1's from 2-, 3- and 4-dimensional binary images. In Discrete Geometry for Computer Imagery, volume 1347 of Lecture Notes in Computer Science, pages 3-18. Springer, 1997.

[19] T. Y. Kong. Minimal non-simple and minimal noncosimple sets in binary images on cell complexes. In Discrete Geometry for Computer Imagery, volume 4245 of Lecture Notes in Computer Science, pages 169-188. Springer, 2006.

[20] T. Y. Kong. Minimal non-deletable and minimal noncodeletable sets in binary images. Theoretical Computer Science, 406(1-2):97-118, 2008.
[21] T. Y. Kong and C-J. Gau. Minimal non-simple sets in 4-dimensional binary images with (8-80)-adjacency. In International Workshop on Combinatorial Image Analysis, pages 318-333, 2004.

[22] T. Y. Kong and A. Rosenfeld. Digital topology: introduction and survey. Computer Vision, Graphics and Image Processing, 48:357-393, 1989.

[23] T.Y. Kong. On the problem of determining whether a parallel reduction operator for $\mathrm{n}$-dimensional binary images always preserves topology. In procs. SPIE Vision Geometry II, volume 2060, pages 69-77, 1993.

[24] T.Y. Kong, R. Litherland, and A. Rosenfeld. Problems in the topology of binary digital images. In Open problems in topology, pages 376-385. Elsevier, 1990.

[25] V.A. Kovalevsky. Finite topology as applied to image analysis. Computer Vision, Graphics and Image Processing, 46:141-161, 1989.

[26] C. Lohou and G. Bertrand. A 3D 12-subiteration thinning algorithm based on P-simple points. Discrete Applied Mathematics, 139:171-195, 2004.

[27] C. Lohou and G. Bertrand. A 3D 6-subiteration curve thinning algorithm based on P-simple points. Discrete Applied Mathematics, 151:198-228, 2005.

[28] C.M. Ma. On topology preservation in 3d thinning. Computer Vision, Graphics and Image Processing, 59(3):328-339, May 1994.

[29] A. Manzanera, T.M. Bernard, F. Prêteux, and B. Longuet. N-dimensional skeletonization: a unified mathematical framework. Journal of Electronic Imaging, 11(1):25-37, 2002.

[30] C.R.F. Maunder. Algebraic topology. Dover, 1996.

[31] C. Ronse. Minimal test patterns for connectivity preservation in parallel thinning algorithms for binary digital images. Discrete Applied Mathematics, 21(1):67-79, 1988.

[32] A. Rosenfeld. A characterization of parallel thinning algorithms. Information and Control, 29:286-291, 1975.

[33] J.H.C. Whitehead. Simplicial spaces, nuclei and $m$ groups. Proceedings of the London Mathematical Society, 45(2):243-327, 1939.

[34] E.C. Zeeman. On the dunce hat. Topology, 2:341-358, 1964. 